\title{
Reuniones virtuales: Adobe Connect y WebEx
}

\author{
Por Jordi Grau-Moracho
}

\begin{abstract}
Resumen: Las videoconferencias, y de forma más amplia, las herramientas de colaboración, llevan años funcionando en internet con mejor o peor fortuna, pero recientemente las grandes empresas del sector de software (Adobe), hardware (Cisco Systems) e internet (Google) están comenzando a tomar posiciones en este nicho del mercado tecnológico, aún muy poco estandarizado y con grandes expectativas de futuro. Se presentan dos de ellas: Adobe Connect y WebEx.
\end{abstract}

Palabras clave: Multimedia, Videoconferencia, Reuniones virtuales, Telepresencia, Colaboración, Asistencia remota, Adobe connect, WebEx.

\section{Title: Virtual meetings: Adobe Connect and WebEx}

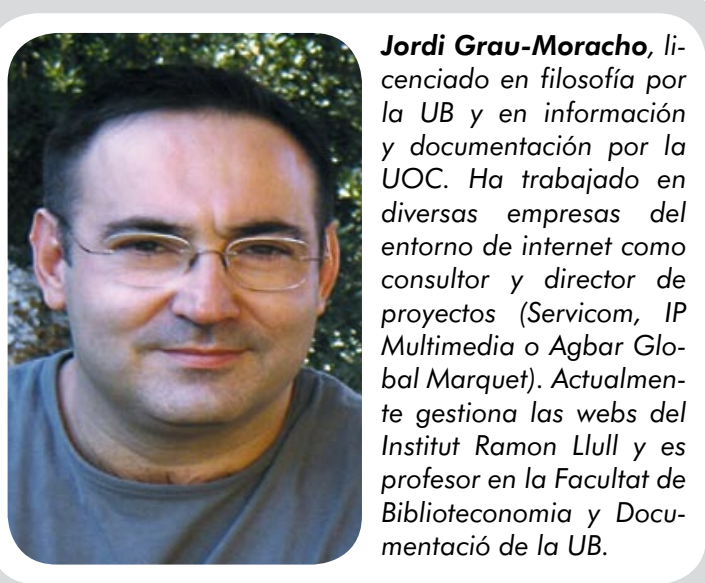

Jordi Grau-Moracho, licenciado en filosofía po UOC. Ha trabajado en diversas empresas del entorno de internet como consultor y director de proyectos (Servicom, IP Multimedia o Agbar Global Marquet). Actualmente gestiona las webs del Institut Ramon Llull y es mentació de la UB.

\begin{abstract}
Videoconferencing and, in a broader sense, collaborative work platforms, have been in use for years, with more or less success. Recently, large companies such as Adobe in the software sector, Cisco Systems in hardware, and Google have begun to enter this niche in the technology market, which is still not standardised and has excellent prospects for the future. Two of these products are discussed: Adobe Connect and WebEx.
\end{abstract}

Keywords: Multimedia, Videoconferencing, Virtual meetings, Collaboration, Remote participation, Adobe Connect, We$b E x$.

Grau-Moracho, Jordi. "Reuniones virtuales: Adobe Connect y WebEx". En: El profesional de la información, 2007, septiembre-octubre, v. 16, n. 5, pp. 518-522.

DOI: 10.3145/epi.2007.sep.16

\section{Introducción}

EN EL AÑO 2010 EL $75 \%$ DE LOS USUARIOS CORPORATIVOS tendrán acceso a las videoconferencias y otras herramientas de trabajo compartido, al igual que ahora usan la web o el correo electrónico, según una previsión reciente de Gartner (Man, 2007). Ante esta perspectiva muchas compañías están tomando posiciones: Adobe, con su Adobe Connect, Cisco Systems acaba de comprar WebEx, y Google ha adquirido Marratech. Y no podemos olvidar el éxito de Skipe, que ya incorpora vídeo.

http://www.adobe.com/es/products/connect/

http://www.ciscosystems.com

http://www.marratech.com/

http://www.webex.es/

Las videoconferencias y los sistemas de colaboración en in- ternet hace ya bastantes años que funcionan con mayor o menor fortuna. Desde Net Meeting de Microsoft o infinidad de programas cliente de colaboración que incluían videoconferencia (o viceversa, programas de videoconferencia que poseían funcionalidades de colaboración) hasta las últimas aplicaciones web, las cosas han cambiado mucho. Y la principal diferencia es que precisamente ahora ya no hace falta disponer de

\section{"Ahora ya no hace falta disponer de ningún software específico que requiera una engorrosa instalación y configuración"}

ningún software específico que requiera una engorrosa instalación y configuración.

$\mathrm{Si}$ se hace un rápido repaso a los programas de colaboración, telepresencia o videoconferencia existentes, se observa una gran dispersión, lo que en cierta forma revela la inexistencia de un estándar dominante: Adobe Connect, WebEx, MeetMeNow, Citrix, Microsoft Office Live Meeting, Netviewer one2meet y Marratech, entre otros muchos.

En este artículo nos centraremos en dos productos que destacan por encima de los demás si atendemos a elementos como la comodidad de uso, pocos requerimientos de software, hardware y red, y numerosas funcionalidades: WebEx y Adobe Connect (derivado de Adobe Breeze). Ambos son multiplataforma al trabajar a través de entorno web. 


\section{Software: el navegador web}

Los dos programas citados destacan por requerir sólo del navegador web (más los periféricos oportunos: cámara, micrófono y altavoces o cascos). En el caso de Adobe todo el software de colaboración corre con una aplicación de Macromedia Flash que se descarga en el momento de acceder al servicio, y con WebEx se descarga una aplicación ActiveX o Java, dependiendo del sistema operativo. Así, sólo hay que disponer del usuario y la contraseña del servicio para poder conectar desde cualquier estación con red (trabajo, casa, hotel o cibercafé).

Otras herramientas para realizar reuniones virtuales requieren de aplicaciones cliente que deben ser instaladas previamente (lo que puede excluir determinados sistemas operativos) y necesitan una cuenta de administrador en el ordenador para instalar y configurar el paquete de aplicaciones (muchas empresas o administraciones no dan este acceso al usuario, para poder controlar el paquete de programas instalados en su red).

En otros casos existen alternativas de software de videoconferencia que requieren configurar la red con unas determinadas especificaciones y sólo permiten una conexión si el ordenador del usuario dispone de una Ip pública (algo bastante difícil si se accede desde una web corporativa, donde muchas Ips son internas) y demandan la apertura de determinados puertos en los firewa$l l s$ de la organización, con el consiguiente problema que eso acostumbra a generar en los departamentos informáticos.

La solución de trabajar a través del entorno web simplifica toda la instalación. El usuario sólo debe disponer de un acceso al servidor de colaboración, o ni siquiera eso si es invitado. Un usuario con cuenta en estos servicios de videoconfe-

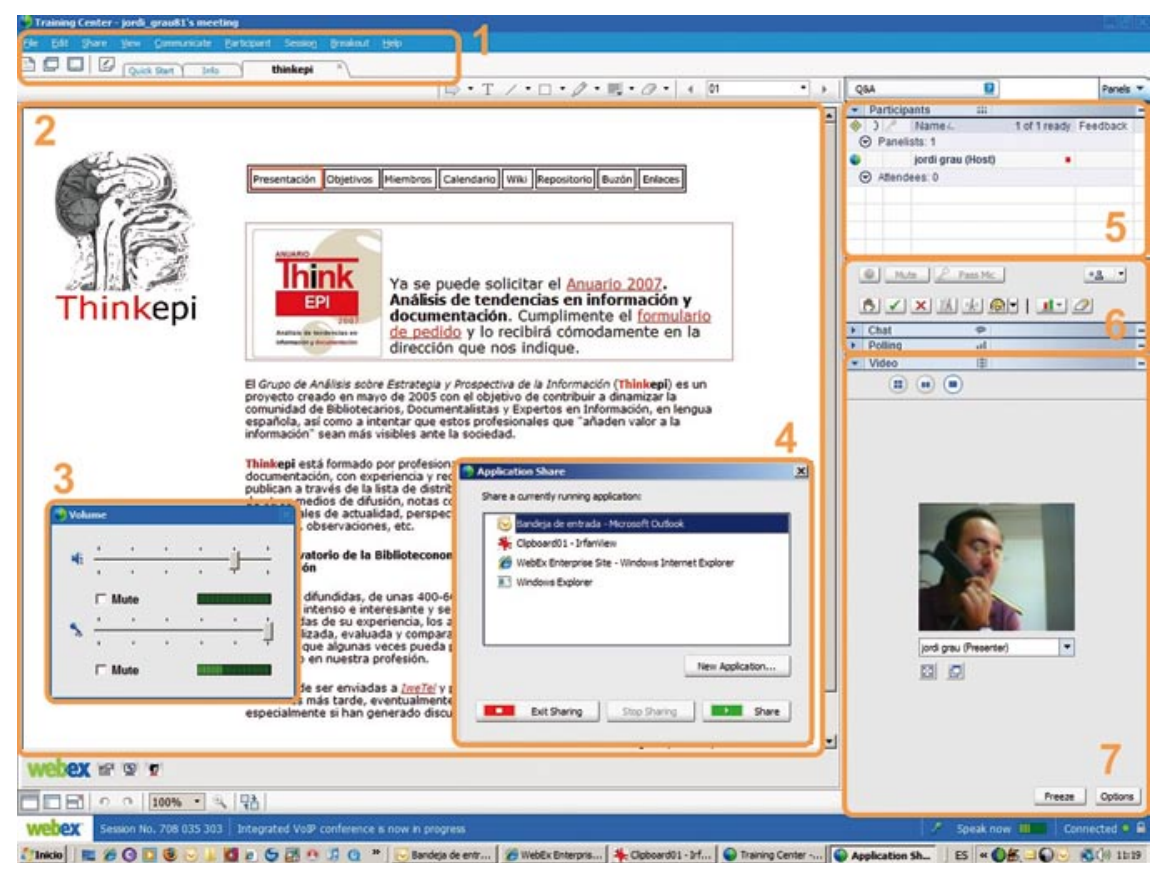

Figura 1. Vista general de WebEx: 1.- Menú de la aplicación 2.- Pagina de web compartida 3.- Control de volumen del altavoz y el micrófono 4.- Herramienta para compartir aplicaciones 5.- Lista de asistentes 6.- Otros paneles colapsados, como encuestas o chat 7.- Panel de videoconferencias

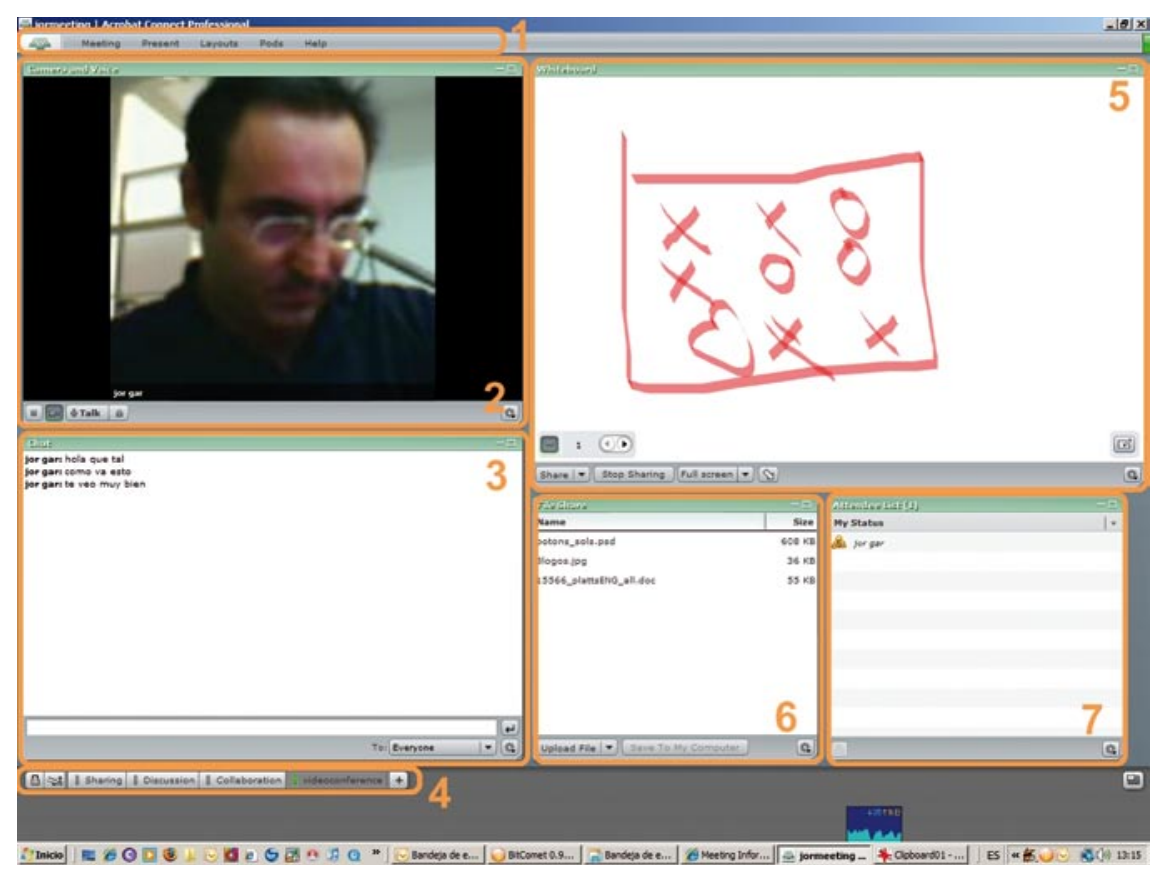

Figura 2. Vista general de Adobe Connect: 1.- Menú de la aplicación 2.- Panel de videoconferencia 3.- Panel de chat 4.- Menú para cambiar la disposición de los paneles (previamente configurados) 5.- Pizarra 6.- Archivos compartidos 7.- Lista de asistentes

rencia puede invitar a sus reuniones virtuales a otros ajenos con sólo enviarles un correo electrónico en donde ya está descrito el motivo de la reunión, la hora y el enlace que deberá clicar para acceder a la sala virtual. Este evento puede quedar insertado en la agenda del Outlook como una reunión más, con el enlace correspondiente a la sala virtual de reuniones.

Las dos plataformas analizadas presentan funciones similares, aunque tienen algunas diferencias respecto al interfaz y a la comercialización. Adobe Connect puede ad- 


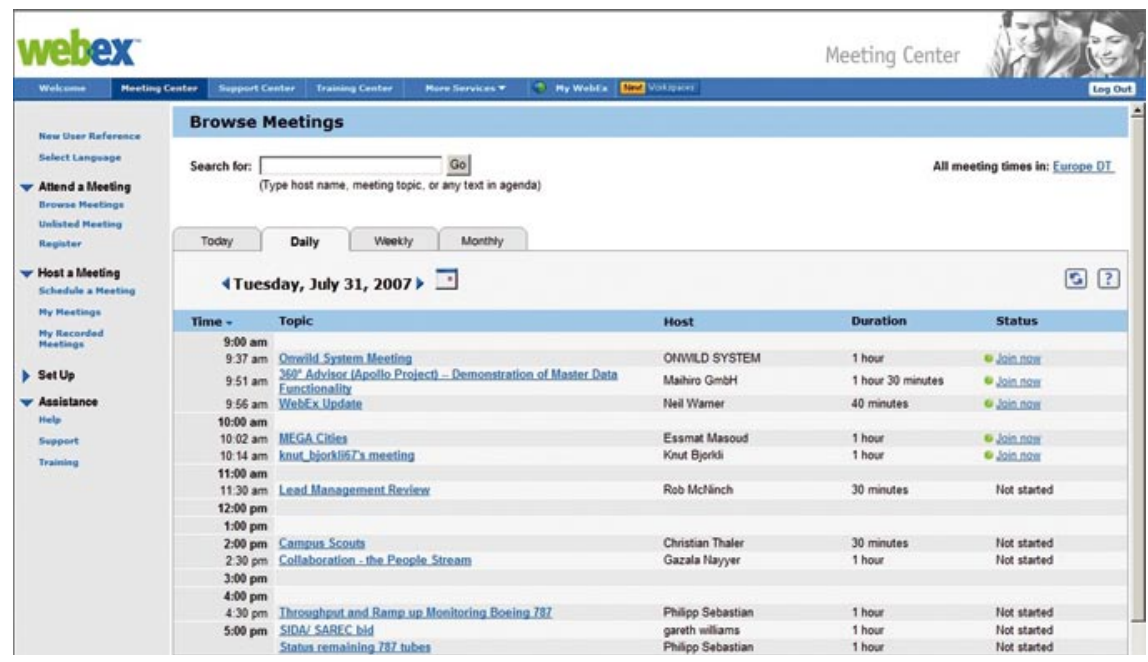

Figura 3. Meeting Center de WebEx, donde se listan las reuniones, convocatorias a eventos y presentaciones, a modo de agenda

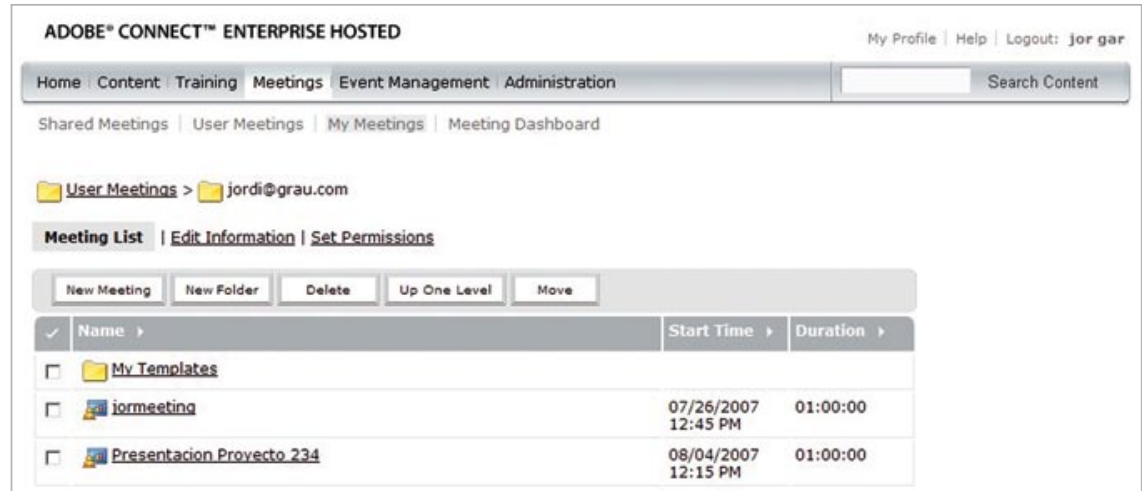

Copyright 92001 - 2007 Adobe Macromedia Software LLC and its licensors. All rights reserved.

Figura 4. Agenda de meetings de Adobe Connect

quirirse como software propietario para ser instalado en los servidores e infraestructura de red del cliente, o como servicio ASP (proveedor de servicios de aplicaciones, en inglés application service provider), proporcionado por los servidores habilitados para ello de Adobe, que dan cobijo a cientos de compañías. WebEx sólo está disponible en esta segunda opción.

Ambas compañías ofrecen varias configuraciones de paquetes de usuarios orientados a distintos segmentos del mercado y con precios diversos. WebEx ofrece además diferentes interfaces de usuario (con alguna funcionalidad diferente en cada caso) para orientarse a múltiples nichos de mercado: Meeting Center (de carácter general), de la reunión: cámaras, voz, contraseñas, privilegios, etc.

Cada asistente recibe su convocatoria y en el momento en que se inicie la reunión, si se ha insertado en su agenda, le aparecerá el aviso. Los asistentes no tienen porque estar registrados en el servicio ya que hay determinados tipos de cuentas que permiten invitar a usuarios externos. Como se ha dicho, ese usuario ajeno al servicio sólo debe disponer de los periféricos adecuados, conexión a internet y un navegador web. El software para realizar la reunión virtual se le cargará en el momento del acceso.

La "sala de reuniones" tiene varios componentes que agrupan las funcionalidades que ya históricamente se han asociado con los entornos de colaboración y videoconferencia: chat, área de compartición de documentos, área de ficheros, pizarra común, comunicación por vídeo y voz, publicación de cuestionarios o encuestas, visualización y control de aplicaciones, gestión de los perfiles de los usuarios, entre las más destacadas.

Las herramientas en ambas plataformas se presentan en ventanas o paneles que se pueden redimensionar o pasar a pantalla completa, dependiendo de la utilidad que se está ejecutando en ese momento. Así por ejemplo, el panel de la imagen de la cámara de vídeo no es muy aconsejable pasarlo a pantalla completa, dada la relativa escasa calidad que ofrece, pero en cambio es muy útil pasar a un panel grande o ventana cuando ejecutamos una aplicación de demostración, hacemos una presentación en PowerPoint o tomamos el control del ordenador de algún asistente para darle soporte remoto. En el caso de Adobe, además pueden guardarse las distintas configuraciones de paneles o escritorios para utilizar en otra ocasión. WebEx por su parte, trabaja con paneles que se desplazan y ventanas flotantes que en algunos momentos 
pueden ser un poco engorrosas de manejar.

Cada panel o ventana gestiona una utilidad, que en ambos programas también son accesibles a través del menú. Así existe un panel para el chat, otro para los documentos compartidos, otro para la imagen de la videoconferencia, etc., y la suma de todos o algunos de ellos componen la pantalla del usuario.

Es destacable la posibilidad de compartir no sólo documentos sino también ventanas de ordenador, aplicaciones o el mismo sistema operativo. Con esto un usuario puede interactuar con el ordenador de otro. El proceso es simple: un usuario comparte un programa en la reunión virtual y todos los integrantes del evento pueden ver la ejecución del mismo. En ese momento cualquier asistente puede pedirle el control remoto de esa aplicación, apareciendo una alerta en la pantalla del usuario propietario, que puede elegir si acepar o rechazar la petición.

También se realizan presentaciones en las que el moderador o presentador va indicando con marcas (lápiz, cuadros, flechas, etcétera) los puntos a destacar.

Dependiendo del tipo de evento (reunión, formación, demostración o soporte técnico) cambia el planteamiento y configuración de la reunión. En unos casos el moderador o presentador tiene todos los privilegios y el resto de los asistentes sólo miran, hasta que llegue el turno de preguntas, donde se les permitirá voz, la pizarra o exponer sus documentos. En otros, el punto fuerte será la participación de todos los asistentes, con lo que el moderador deberá dar la palabra o quitarla siguiendo los criterios que se hayan establecido.

Si se trata de un servicio de soporte técnico, que es más específico (solicitar una ayuda a un especialista sobre una aplicación concreta),

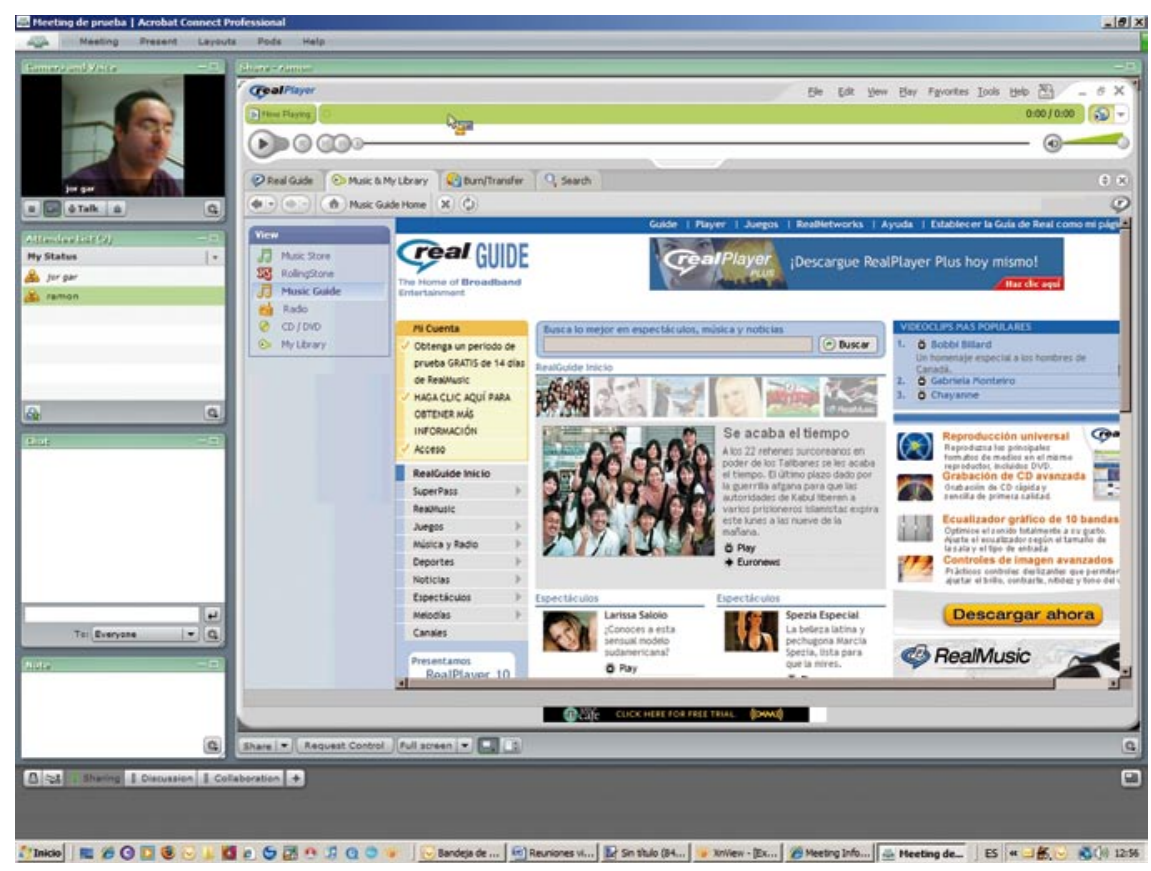

Figura 5. Un usuario comparte la aplicación RealPlayer con los asistentes, que pueden llegar a controlar remotamente si el usuario propietario les da acceso

el usuario llama al Call Center de atención para solicitar la ayuda de un técnico. Éste puede solucionar el problema con el chat, la voz o indicar en su propio ordenador cual es método para superarlo. Pero si persiste el problema, el entorno permite que el técnico tome el control del ordenador del usuario para configurar o ejecutar la solución más adecuada.

\section{Componente crítico: la voz}

El rendimiento de ambos entornos es aceptable siempre que dispongamos de un ancho de banda dedicado. En ese sentido, las redes corporativas adolecen de más problemas que las conexiones residenciales, dado que hay momentos de la jornada en la que la conexión a internet puede estar más saturada. La conexión particular por $A D S L$ de un megabyte acostumbre a ser suficiente y el usuario tiene todo el control sobre los usos de la misma.

En las pruebas realizadas en tres escenarios distintos: un $A D S L$ residencial de un megabyte, un $A D S L$ corporativo de 4 megabytes (poco saturado) y una red corporativa en la que trabajan más de 80 personas (intermitentemente algo colapsado); el resultado ha sido relativamente óptimo tanto con Adobe como con WebEx, aunque en algunos momentos la voz se ha oído entrecortada, lo que ha obligado a pasar a la opción de chat o finalizar la reunión.

Hay que tener en cuenta que la voz es crítica en las reuniones virtuales. Si la imagen de vídeo se ralentiza o baja de calidad durante el evento no supone un gran problema para los asistentes, que pueden mantener la atención en la voz del conferenciante, las presentaciones o la pizarra. Pero si la voz se pierde o entrecorta es casi imposible mantener el hilo de la presentación o conversación.

WebEx aporta una solución externa a internet para solventar este problema a través de una red de centralitas conectadas en los principales países para poder realizar una multiconferencia telefónica a precio de llamada local para cada asistente. La llamada telefónica se combina con el software de colaboración, en el que se excluye la voz Ip. Esta solución también permite que en la misma reunión haya usua- 


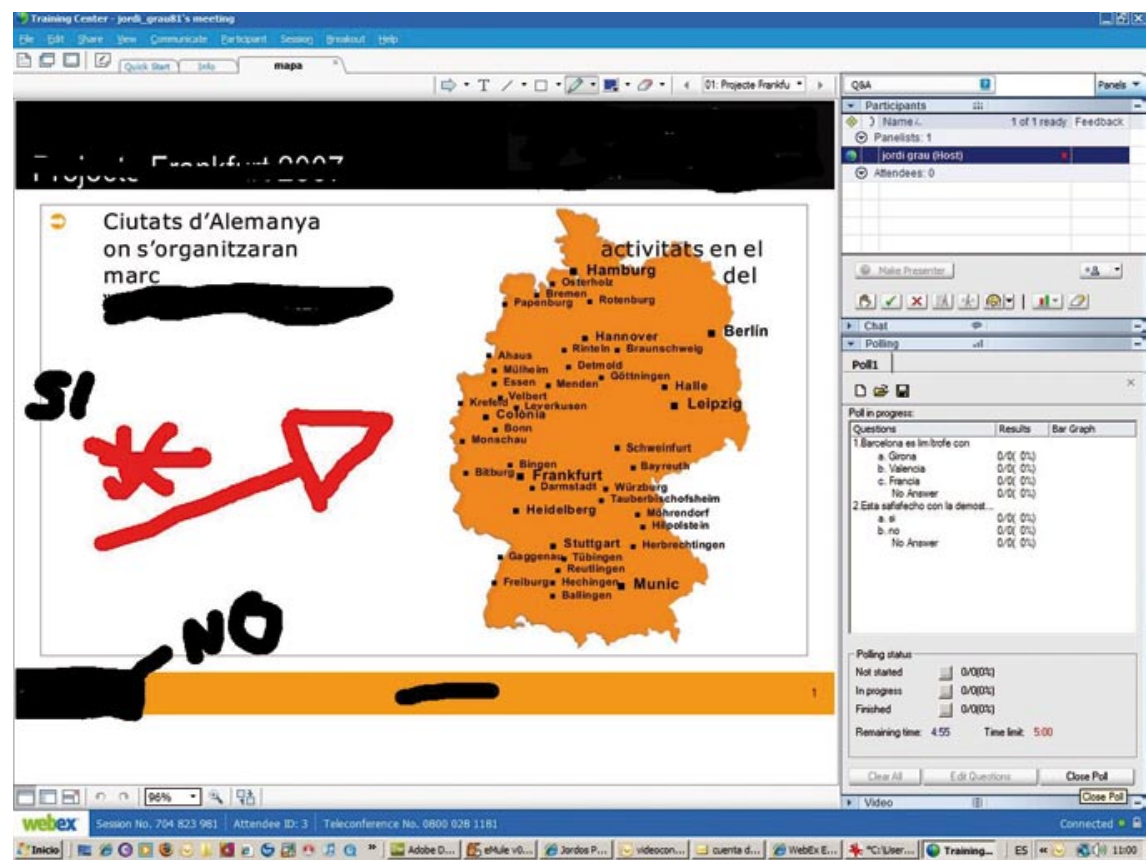

Figura 6. Presentación sobre la que se están realizando modificaciones con la pizarra. A la derecha se puede ver el panel de encuestas.

rios conectados al teléfono y otros por voz Ip.

En el caso de multiconferencias por voz Ip es aconsejable que los participantes liberen a la conexión de internet de aplicaciones que ocupan un ancho de banda de forma permanente o elijan momentos en que su red corporativa va menos cargada.

El control remoto de aplicaciones $\mathrm{u}$ ordenadores demanda también de un ancho de banda continuo y mantenido. Si la conexión se satura en el momento en que un participante controla el ordenador o un programa remotamente, se puede caer la aplicación de colaboración, aunque en ninguna ocasión se haya producido la caída del ordenador.

El resto de utilidades: presentaciones, compartición de páginas web, documentos o pizarra han funcionado satisfactoriamente en la totalidad de las pruebas realizadas. Seguramente debido a que la transferencia de información de un ordenador a otro puede tardar más o menos, pero una vez transmitida ya está a disposición de los asistentes como si se tratara del tiempo de carga de una página web.

Adobe Connect y WebEx aun funcionando razonablemente bien en ordenadores de tipo medio con una conexión corporativa y periféricos de gama baja, requieren ciertas condiciones para obtener un resultado óptimo. Una cámara web de calidad y un micrófono que elimine el ruido de fondo mejorarán sustancialmente la calidad de la videoconferencia. Una iluminación adecuada en las salas físicas donde se realicen las reuniones y un cierto aislamiento de los ruidos externos al evento facilitará la atención de los asistentes sobre los presentadores. El ancho de banda también es muy importante, ya que de existir fluctuaciones durante la reunión puede provocar que la compartición de documentos o aplicaciones se corte durante algunos segundos, perdiéndose el hilo argumental del discurso o presentación que se mostraba en pantalla.

A la hora de plantearse la inmersión en reuniones virtuales hay que tener presente que este tipo de herramientas requieren una cierta práctica para poder aprovechar todas sus posibilidades, y que es necesario cierta firmeza para luchar contra hábitos tan arraigados como la reunión presencial o la llamada telefónica. Por ello es recomendable realizar una implantación progresiva, con objetivos a largo plazo.

\section{Bibliografía}

Expansion.com. "Google compra Postini para potenciar su área de e-mails". 09-07-07. Consultado en: 28-07-2007.

http://www.expansion.com/edicion/exp/empresas/tecnologia/es/desarrollo/1014546.html

Grau-Moracho, Jordi. "Reflexión abierta sobre las reuniones virtuales en Internet". En: ThinkEpi, 27-07-07.

http://www.thinkepi.net/repositorio/reflexionabierta-sobre-las-reuniones-virtuales-en-internet/

Man, Jeffrey. "Magic quadrant for web conferencing, 2007". En: Gartner RAS Core research NOTE G00147406, 1 May 2007, R2340, 06202008. Consultado en: 28-07-07.

http://www.adobe.com/enterprise/pdfs/magic quadrant_web_conferencing2007.pdf

Jordi Grau-Moracho, Institut Ramon Llull.

jgrau@llull.cat

\section{¿Recibes ya EPI en casa?}

Por sólo 75 euros $+4 \%$ IVA

puedes tener tu copia particular y leerla cómodamente cuando quieras. 